\title{
Heat shock proteins in microbiologia clinica
}

\section{Orazio Andreoni}

già Primario del Laboratorio di Microbiologia e Virologia, Ospedale Maggiore della Carità di Novara

\section{RIASSUNTO}

In questa breve rassegna si è cercato di valutare la posizione in microbiologia clinica di particolari proteine, che oltre a concorrere nel ripiegamento di catene oligomeriche impedendone l'aggregazione (chaperones), si traducono in Heat shock proteine o stress proteine di fronte ad un aumento di temperatura o di altre improvvise modificazioni ambientali. Definita la loro sintesi per l'intervento di specifici fattori sigma, sono stati riportati esempi di Hsp-omologia tra specie batteriche diverse, nonché la loro possibilità di agire come antigeni o come fattori di virulenza. Stante l'omologia tra talune Hsp umane e Hsp di origine batterica, per alcune forme morbose soprattutto ad andamento cronico, è stata brevemente riesaminata una loro possibile correlazione con processi di autoimmunità.

\section{SUMMARY}

In this short review we tried to value the position of clinical microbiology regarding particular proteins called chaperones. These proteins complete the folding on protein chain and prevent their aggregation. Because of increase in temperature or other sudden environment variations they are called Heat shock proteins or stress proteins. They play an important role for the specific s factor, but actually they have also other functions: Hsp homology between different species of bacteria; their antigenic activity and their role in being factor of virulence. Overall in the chronic pathology, finally we find out their possible involving in the autoimmunity process based on the proving of homology between human and bacteria Hsp.

Prospettata la loro esistenza a seguito di riscontri genetici in cromosomi di ghiandole salivari di larva di Drosophyla melanogaster (35) e successivamente definite come tali (40), si tratta di molecole che possono essere indotte in tutte le cellule eucariote e procariote, quali probabile espressione di costanti biologiche nella evoluzione cellulare. Distinte in varie famiglie in base al peso molecolare, di cui ai prototipi Hsp100, Hsp90, Hsp70, Hsp60, Hsp40, Hsp10 e localizzate in vari scompartimenti intracellulari (citoplasma, mitocondri, reticolo endoplasmatico), in condizioni fisiologiche possono agire come chaperones, ossia come molecole responsabili dell'assemblaggio, ripiegamento e traslocazione di proteine oligomeriche (9, 34) (figura I). Sensibili ad aumenti di temperatura e, comunque ad interventi stressanti (deficienze nutrizionali, radiazioni ultraviolette, agenti chimici, infezioni varie) tanto che la stessa dizione Heat shock proteins è stato talora sostituita da stress shock proteins, la loro concentrazione intracellulare può andare incontro a sensibili cambiamenti in rapporto all'intensità del variare delle condizioni ambientali.

In microbiologia, il tema Hsp, trattato a livello di numerose specie patogene, soprattutto a replicazione intracellulare, si presta ad una considerevole serie di osservazioni, con taluni specifici riferimenti ai processi di sintesi, alle omologie tra singole specie, alle proprietà antigeniche, ad espressioni di patogenicità e, nel contesto clinico, a manifestazioni di autoimmunità.

\section{SINTESI E SWITCHING TRASCRIZIONALE}

A livello cellulare l'induzione di Heat shock proteine è sottoposta alla regolazione trascrizionale da parte del fattore sigma, che consente appunto alla cellula di adattarsi alle svariate condizioni

FAMIGLIA

SEDE INTRACELLULARE

FUNZIONE

\begin{tabular}{ccc}
\hline Hspl0 & Citoplasma, nucleo & Stabilizzazione citoscheletro \\
\hline Hsp40 & Citoplasma, nucleo, reticolo endoplasmatico & Regola attività Hsp70; lega proteine non native \\
\hline Hsp60 & Mitocondri, citoplasma & $\begin{array}{c}\text { Lega polipeptidi parzialmente ripiegati e } \\
\text { controlla ripiegamento }\end{array}$ \\
\hline Hsp70 & $\begin{array}{c}\text { Citoplasma, nucleo, mitocondri, } \\
\text { reticolo endoplasmatico }\end{array}$ & $\begin{array}{c}\text { Lega polipeptidi estesi e previene } \\
\text { l'aggregazione di peptidi non ripiegati }\end{array}$ \\
\hline Hsp90 & Citoplasma, reticolo endoplasmatico & $\begin{array}{c}\text { Regola l'attività di proteine e previene } \\
\text { l'aggregazione di proteine ripiegate }\end{array}$ \\
\hline Hspll0 & Citoplasma, nucleolo & Ripiegamento proteico, tolleranza termica \\
\hline
\end{tabular}

Figura I. Classificazione, sede e funzione di $\operatorname{Hsp}$ (34, ridotta) 
ambientali. In batteri Gram-negativi, come Escherichia coli, dove DnaK ha circa il $50 \%$ di identicità con Hsp70 di cellule eucariote e GroEL corrisponde a Hsp60, oltre al fattore primario sigma 70 , il più abbondante nella fase esponenziale di crescita batterica, esistono altri fattori alternativi capaci di promuovere l'espressione di diversi set di geni a seconda del variare delle condizioni ambientali. Tra questi, sigma 32, sensibile all'aumento della temperatura, concorre in primo piano nella trascrizione di geni heat shock, venendo poi sequestrato da DnaK chaperone e degradato da proteasi ATP dipendenti (21) (figura II). Lo stesso potrebbe valere per $\sigma^{\mathrm{B}}$ in grado di rispondere coordinatamente ad una varietà di segnali chimici e fisici e di facilitare la crescita di Listeria monocytogenes in condizioni di elevata pressione osmotica (4). Nei batteri Gram-positivi, come Bacillus subtilis la regolazione di geni shock, groE e dnaK, comporta l'intervento di sequenze specifiche definite CIRCE (controlling inverted repeat chaperone expression).

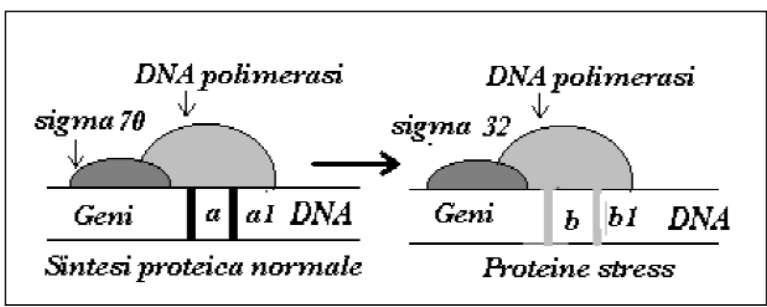

Figura II. Switching del fattore sigma per la sintesi di proteine stress (2I)

\section{OMOLOGIA INTERCELLULARE SPECIE-SPECIFICA}

Uno degli assunti più rilevanti nella biologia di Hsp si riferisce all'alto grado di omologia, circa il $50 \%$, tra i membri di una stessa famiglia derivati da cellule eucariote e procariote. Ad esempio, DnaK di Campylobacter jejuni è risultato omologo tra il 53 e il $78 \%$ a quello di altre specie batteriche (39) (figura III), così come, dopo il riscontro di un comune antigene con reattività crociata tra Legionella pneumophila e Pseudomonas aeruginosa, sequenze di GroEL e GroES di quest'ultima specie hanno esibito il $78 \%$ di omologia con quelle di Hsp10 e Hsp60 di altre specie (36). Di interesse del tutto particolare la dimostrazione che, a seguito di "trapianto", Hsp60-Hsp10, ottenuti da mitocondri umani sono stati in grado di poter sostituire completamente GroEL e GroES di Escherichia coli, restaurandone le specifiche proprietà metaboliche (31). Sottoposta ad un aumento termico, in pochi minuti Neisseria gonorrhoeae sintetizza Hsp in grado di reagire immunologicamente con DnaK e GroEL di Escherichia coli (42), così come le proteine Stp11 e Sta58 di Rickettsia tsutsugamushi, intesa la seconda come il maggior antigene del batterio, presentano una assai elevata omologia con GroES e GroEL, sempre di Escherichia coli, pur differenziandosi per proprietà antigeniche (38). In Helicobacter pylo$r i$, la proteina $\mathrm{HspB}$, che funziona come chaperone molecolare per ureasi e che con questo enzima è localizzato nel citoplasma batterico nel corso della fase logaritmica di crescita per localizzarsi a livello di membrana nei processi di autolisi (33), corrisponde a geni che codificano per un omologo di GroES di altre specie batteriche e per Hsp umane (13). In Chlamydia.trachomatis, dopo la dimostrazione che una proteina di $40 \mathrm{kDa}$ è presente nella membrana esterna dei corpi reticolati, così come lo è una proteina di $75 \mathrm{kDa}$ con sequenza in aminoacidi in gran parte sovrapponibile a quella di Hsp70 di Escherichia coli e Bacillus megaterium (10), è stato isolato un gene che codifica per due tipi di proteine, di cui una, di $57 \mathrm{kDa}$, presenta una sequenza in aminoacidi con un alto grado di omologia con le sequenze Hsp60 umane e di numerose specie batteriche (8). In Legionella pneumophila, lo studio di sequenze di aminoacidi ha messo in evidenza che la proteina HtpB esibisce un alto grado di omologia con $65-\mathrm{Kda} d i$ Mycobacterium tuberculosis, con GroEL di Escherichia coli e HtpB di Coxiella burnetii (19) (figura IV). Lo stesso vale per Ehrlichia sennetsu, dove le sequenze in aminoacidi di Hsp70 sono risultate omologhe a quelle di parecchie specie batteriche (46) (figura V). È stato fatto osservare, tuttavia, che Heat shock proteine della stessa famiglia, ma di specie diverse, possono espletare funzioni diversificate: Mycobacterium tuberculosis, ad esempio, contiene geni multipli che codificano per Hsp60, ma, pur presentando una grande similarità di aminoacidi, alcuni cloni esprimono una attività $10-100$ volte maggiore di quella di altri (25). Nell'ambito di specie di uno stesso genere, infine, come Staphylococcus capitis, cohnii, schleiferi, l'omologia di sequenze in Hsp60 si presenta in percentuali ancora più elevati, pari al 91-98\%, così come scarsa microeterogeneicità contraddistingue Hsp60 gene di stipiti diversi di Staphylococcus aureus (22).

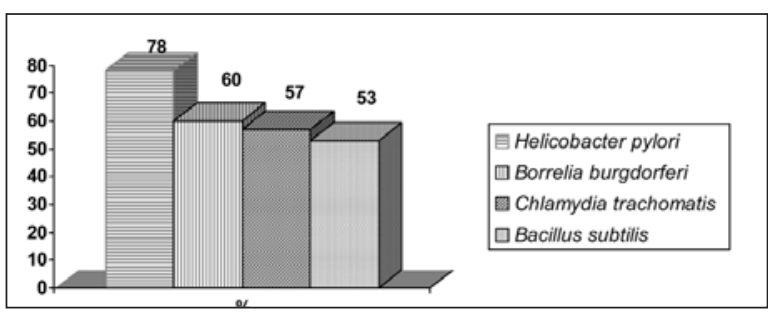

Figura III. Omologie di DnaK (Hsp70) di Campylobacter jejuni (39) 


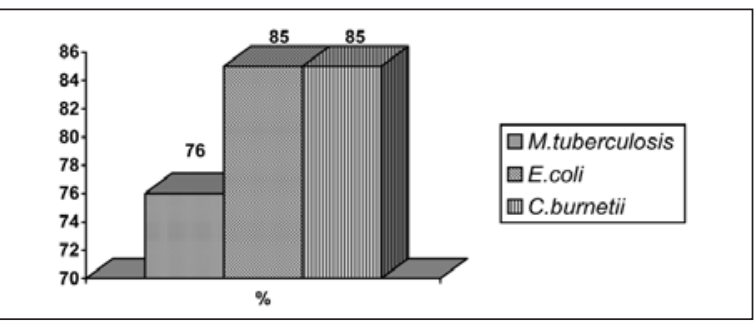

Figura IV. Omologia della proteina $\mathrm{HtpB}$ di Legionella pneumophila (19)

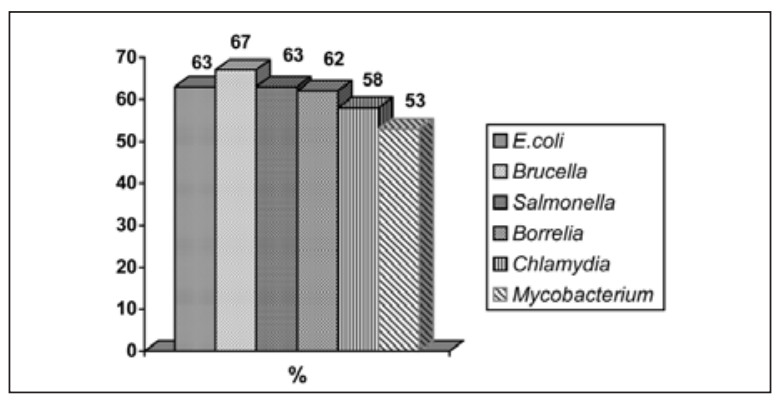

Figura V. Omologia di aminoacidi di Hsp70 di Ehrlichia sennetsu (46)

\section{HSP COME ANTIGENI}

Nell'ambito di Hsp, Il prototipo è GroEL di Escherichia coli, il cui ruolo biochimico è quello di chaperone molecolare in quanto aiuta il ripiegamento e assemblaggio di catene polipeptidiche, impedendo così la formazione di intermediari irregolari. La proteina è costituita da numerose subunità con epitopi simili a quelli di altri membri della famiglia Hsp60 ritenuti potenti immunogeni in infezioni sostenute da varie specie di batteri (M. tuberculosis, M. leprae, C. trachomatis). In qualità di antigeni sarebbero coinvolti nella sopravvivenza batterica nell'ospite, nella patogenesi della forma morbosa e nella induzione di autoimmunità. In particolare, per quanto riguarda Mycobacterium tuberculosis, questa specie può esprimere un serie di Hsp di elevato peso molecolare, di cui GroEL presenta almeno 12 diversi epitopi e i cui anticorpi specifici reagiscono con proteine di altre specie di micobatteri. Un'altra Hsp di basso peso molecolare, GroES o Hsp10, è stata descritta come il maggiore $\mathrm{T}$ antigene riconosciuto da soggetti malati e, sempre in questi ultimi, avrebbe la proprietà di indurre la produzione di alti livelli di IFN- $\gamma$. Più specificatamente, in rapporto alle variazioni di temperatura, è stato visto che se questa aumenta da $37^{\circ} \mathrm{C}$ a $42^{\circ} \mathrm{C}$ si realizza una aumentata sintesi delle tre proteine maggiori, mentre, di fronte un ulteriore aumento, queste ultime diminuiscono comparendone altre di basso peso molecolare (Hsp20, Hsp15) (44).

La presenza di anticorpi specifici verso Hsp è stata rilevata in diverse forme morbose. In sieri di soggetti con pertosse e di soggetti trattati con vaccino DTP (difterite, tetano, pertosse) sono stati evidenziati anticorpi verso la proteina $63-\mathrm{kDa}$ di Bordetella pertussis (7), mentre un anticorpo monoclonale verso $60-\mathrm{kDa}$ di Legionella pneumophila sarebbe da attribuirsi ad un unico epitope, comune, peraltro, in tutte le 59 specie del genere Legionella, ma, diversamente da altri anticorpi, senza reattività crociata con altre specie batteriche (37). Sempre per quanto riguarda Hsp60, con anticorpi monoclonali non solo sono stati classificati genere e specie di C. trachomatis, ma è stata localizzata la sede di epitopi nelle sequenze carbossiliche e aminoterminali, oppure in specifiche regioni nel caso di positività crociate (45), con il riscontro, inoltre, che sieri di pazienti con alti titoli sono risultati reagire preferibilmente con la parte carbossilica della molecola. Sempre sulla base di rilievi sierologici è stato proposto di ricorrere al riscontro di epitopi Hsp60 a scopo diagnostico e per lo studio dei meccanismi immunopatologici in forme morbose e soprattutto in sequele patologiche post-infettive (8). Lo stesso è valso per Hsp10, repertata con notevole frequenza nella popolazione francese attraverso riscontro di anticorpi specifici e associata con cronicità di infezioni del tratto genitale (6). Verso BaGroEL (Hsp62), infine, ottenuto con Hsp70 e Hsp10 da uno stipite rugoso di Brucella abortus sottoposto tra $42-46^{\circ} \mathrm{C}$, si sarebbe manifestata una risposta immune in bestiame vaccinato con questa specie di patogeno intracellulare, non associata, peraltro, ad effetti protettivi. È stato precisato, inoltre che la proteina, una volta liberata, viene come di norma processata e presentata sulla superficie di macrofagi e successivamente esposta a $\mathrm{T}$ cellule che stimolano una risposta immune (26).

\section{HSP COME EQUIVALENTI DI PATOGENICITÀ}

Nel corso di una infezione, Actinobacillus actinomycetemcomitans, responsabile di malattie periodontali in virtù di accertati fattori di virulenza (leucotossina, emolisina) e di eventi immunosoppressivi (interferenza nella sintesi di IgG e IgM), esposto a modificazioni ambientali, può essere indotto a sintetizzare proteine stress. Tali GroEL o Hsp60, confermata come molecola cilindrica di $12 \mathrm{~nm}$ di diametro, presente in frazioni solubili e nella membrana di batteri sottoposti a $43^{\circ} \mathrm{C}$, provvista di attività citotossica in quanto in grado di aumentare la proliferazione dei legamenti periodontali (17). Per quanto riguarda Hsp60, indotta in altre specie batteriche, è stato rilevato di recente che in Legionella pneumophila questa proteina, diversamente dalla omologa in 
Escherichia coli, ha una collocazione extracitoplasmatica associandosi a proteine di membrana e al lipopolisaccaride, potendo così concorrere nei meccanismi di secrezione e nella patogenesi dell'infezione (16). Sempre in Legionella pneumophila, per stabilire se l'induzione di Hsp60 è correlata con la virulenza, è stato operato un confronto tra stipite virulento e non virulento, evidenziando, in quest'ultimo, un minore coinvolgimento di specifici geni e la mancanza di una risposta stress in sede intracellulare (monociti umani), al contrario di quanto avviene di norma con microrganismi a replicazione intracellulare (15). Un legame, infatti, tra sintesi di proteine stress e sopravvivenza di batteri patogeni è stato indicato con Salmonella typhimurium e Listeria monocytogenes, per la loro capacità, come i micobatteri, di sopravvivere entro fagociti dell'ospite. Una precisa responsabilità di carattere patogenetico è stata riconosciuta comunque a Hsp $66 \mathrm{kDa}$ ottenuta da Salmonella typhimurium nel supernatante a seguito di aumento di temperatura, definita tale in quanto riconosciuta da specifici anticorpi monoclonali e responsabile appunto di aggregare e legare i batteri a muco intestinale (14).

Con l'impiego di linee cellulari (Caco-2) è stato anche osservato che uno stipite di Salmonella enteritidis è in grado di interferire con l'integrità dello strato superficiale degli enterociti e ciò ad opera di un aumentato livello di Hsp70 e Hsp90, mentre l'endotossina non indurrebbe espressioni di shock (29). Di particolare interesse alcune osservazioni a livello di Haemophilus ducreyi, responsabile dell'ulcera molle o cancroide dei genitali, dove, considerata la scarsa disponibilità del batterio a diffondere dalla cute durante l'infezione, è stato possibile dimostrare che alti livelli di GroEL sono coinvolti direttamente o indirettamente nella sopravvivenza e adesività del batterio stesso in presenza di condizioni ambientali stressanti. È stato rilevato anche che la presenza plasmidica di geni per DnaK oltre ad aumentarne la produzione, provoca una diminuzione di GroEL, con la possibilità pertanto che DnaK agisca da modulatore nei confronti di GroEL (32). Sempre in tema, infine, di batteri intracellulari obbligati, in Ehrlichia sennetsu è stata dimostrato che a seguito di un aumento di temperatura il batterio è in grado di operare la sintesi di Hsp 60 e Hsp70, con il prospetto che quest'ultima, presente sulla superficie batterica e con una sequenza di aminoacidi sovrapponibile per il 53-63\% a quella di Hsp70 di batteri del genere Brucella, Borrelia, Chlamydia, Mycobacterium, possa concorrere nella adesione a cellule ospiti (46).

\section{HSP: AUTOIMMUNITÀ, AUTOREATTIVITÀ ED AZIONE ANTINFIAMMATORIA}

Ancora recentemente è stato ribadito che Hsp sono molecole immunodominanti e che gran parte della risposta inmmune nei confronti di batteri patogeni è diretta verso peptidi derivati da queste proteine. In considerazione della similarità fillogenetica tra batteri e gli organismi superiori di queste molecole e la conseguente potenzialità per reazioni crociate, è stato possibile presumere che Hsp possano agire come potenziali autoantigeni e che lo stabilirsi di reazioni crociate rappresenti un legame tra infezione e autoimmunità. In letteratura non mancano riscontri e talune ricerche, condotte nel campo dell'artrite cronica, sclerosi multipla, diabete infantile hanno indicato, infatti, una marcata risposta immune a Hsp60 come possibile legame patogenetico tra infezione batterica e malattia cronica ad impronta autoimmune. Ad esempio, per quanto riguarda il diabete infantile, di recente, in un gruppo di pazienti è stata evidenziata una risposta $T$ cellule e la presenza di autoanticorpi Hsp70 in una percentuale molto più elevata rispetto a quella ottenuta nei controlli (figura VI) (1). Lo stesso è valso nei confronti di un gruppo di pazienti con spondilite anchilosante, dove è emersa una relazione tra forma morbosa $\mathrm{e}$ Hsp60 di batteri del genere Klebsiella, Escherichia, Yersinia, Salmonella (12), mentre non è stato escluso che cellule endoteliali abbiano la possibilità di legarsi ad Hsp60 autologhe o microbiche, quale meccanismo iniziale delle prime fasi di arteriosclerosi (41). Riconosciuto che neurite e danni a livello di nervi sono i più importanti caratteri della lebbra, che l'invasione del nervo è una caratteristica di Mycobacterium leprae, osservazioni istopatologiche e indagini in vitro hanno dimostrato che le cellule Schwann, ossia le cellule gliali del sistema nervoso periferico, sono colpite di preferenza albergando in sede intracellulare una notevole carica batterica. La mancata azione citotossica di questi ultimi ha indotto a ritenere che il danno nervoso sia il risultato di una risposta immunitaria cellulo-mediata, dovuta appunto alla liberazione di citochine (TNF, IFN- $\delta$ ) da parte di macrofagi attivati e ad un attacco diretto di $\mathrm{T}$ linfociti citotossici alle cellule infettate. Successive indagini, hanno prospettato che la patologia nervosa sia condizionata dall'intervento di Hsp70, sintetizzata appunto a livello di cellule Schwann, oppure da Hsp60 la cui sintesi in determinate linee cellulari, come quelle monocitarie, avrebbe un decisivo significato in rapporto alla manifestazione autoimmune $(5,30)$. Premesso che T cellule che riconoscono omologhi di Hsp60 sono state identificate nel sangue perife- 
rico e nel liquido sinoviale in casi di artrite reumatoide e nella malattia di Lyme, in quest'ultimo caso processi autoimmuni sarebbero sostenuti anche da Hsp70 indotte da Borrelia burgdorferi ma non da Hsp70 autologhe (3).

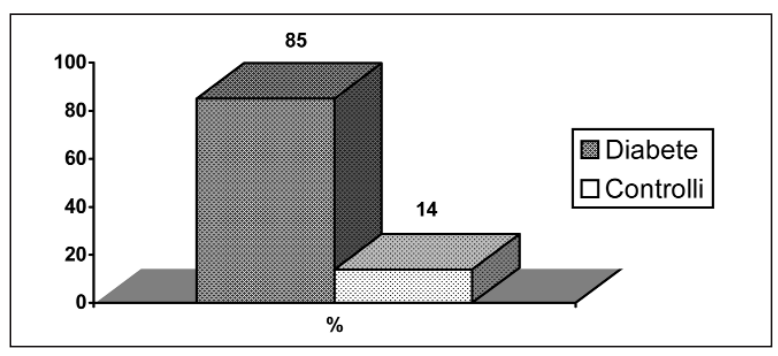

Figura VI. Risposta autoanticorpale a Hsp70 in diabetici e in controlli (I)

Sempre sul piano microbiologico, una specie più volte chiamato in causa è stata Chlamydia trachomatis, parassita intracellulare obbligato, responsabile di svariate forme morbose a livello oculare e urogenitale, che possono progredire in forme infiammatorie croniche con cecità e infertilità. La patofisiologia di questi eventi cronici sarebbe mediata immunologicamente e Hsp60 è stata implicata come il principale antigene per stimolare risposte immunopatologiche, come un'infiammazione mononucleare quando posta sulla superficie congiuntivale di animali già colpiti da una infezione oculare causata da questa specie (45). D'altro canto, ancora recentemente correlazioni tra risposte immuni e gravi sequele da infezioni sostenute da Chlamydia trachomatis sono state dimostrate in donne con malattia infiammatoria pelvica, gravidanza ectopica e in pazienti con artrite (11). Sequele immunopatologiche che troverebbero riscontro nell'alto grado di identicità delle sequenze di Hsp60 e come lo dimostrerebbero i risultati di saggi su animali trattati con Hsp60 proprie e Hsp60 di Chlamydia trachomatis, singole o in contemporaneità e dove una forte proliferazione di $\mathrm{T}$ cellule e alti titoli anticorpali hanno contraddistinto l'esito di più trattamenti con entrambe le proteine (43).

Un'altra specie dello stesso genere, Chlamydia pneumoniae è stata associata a malattie croniche come asma, artrite e arteriosclerosi e ricerche in questa direzione hanno indicato nella eccessiva risposta immune a Hsp60 il legame tra l'infezione batterica e malattia cronica. Stante importanti saggi in casi di sindrome acuta coronarica e di angina stabile, si ritiene che entrambe, Hsp del batterio e Hsp umane possano essere sottoposte a condizioni di stress da reazioni crociate per la formazione di anticorpi citotossici da cui la possibilità che la reazione immune assuma un importante ruolo nella aterosclerosi coronarica
(2). È stato ribadito che in questi casi anche se singoli individui non presentano la stessa sensibilità attraverso meccanismi diversi, il riscontro di Hsp60 in cellule delle placche ateromasiche non può che avere un valido significato interpretativo (23) e che il persistente riscontro di elevati livelli di IgA anticorpi verso Hsp60, soprattutto se associato a valori elevati di proteina $\mathrm{C}$ reattiva, non può che rappresentare un indice prognostico sfavorevole quale rischio coronarico (20). Di rilievo anche il dato relativo a controlli praticati su alcune centinaia di soggetti con lesioni coronariche dove la presenza di elevate anticorpi IgG solo verso Hsp60 di Chlamydia pneumoniae e non verso Hsp di altre specie batteriche e umane (28).

Nel genere Campylobacter, la specie C. jejunii, distinta in 60 sierotipi in base all'antigene $\mathrm{O}$, è responsabile di gravi forme di diarrea, mentre il sierotipo $\mathrm{O} 19$ è stato associato più frequentemente alla sindrome di Guillain-Barré e i sierotipi O2, O10, O23 alla sindrome di Miller Fisher, due neuropatie demielinizzanti del sistema nervoso periferico, ritenute essere mediate da meccanismi autoimmunitari. Sempre per quanto riguarda patologie dell'apparato digerente, è stato fatto presente che parte della popolazione con enteropatia da Salmonella sviluppa una artrite reattiva, mentre l'isolamento del batterio dalla articolazione deporrebbe per un legame tra infezione e autoimmunità una volta riconosciuto un epitope immunodominante nella molecola di GroEL (27).

Per quanto riguarda altre importanti proprietà di Hsp, è stato visto che Hsp60 umane e di sette specie batteriche, tra cui Escherichia coli, Chlamydia pneumoniae, Mycobacterium bovis, sono in grado di determinare una risposta infiammatoria a livello di macrofagi di topo con produzione di TNF- $\alpha$, con il riscontro però che ogni specie è in grado di reagire con questo tipo di cellule attraverso una diversa via (18). Ugualmente, è stato prospettato un quadro di reattività crociata tra Hsp di Helicobacter pylori e gastrite, anche se i reperti istopatologici, quali persistenza di flogosi anche dopo che il batterio è stato eliminato e continua presenza del batterio per mantenere l'infiammazione, non si concilierebbero con uno stato di autoimmunità (24). D'altro canto, come è stato fatto osservare di recente (34), nell'ambito di un evento immunitario, la reattività cellulare (monociti, macrofagi, T cellule) e la produzione di citochine possono differenziarsi in processi proinfiammatori e antinfiammatori con diversa affinità verso proteine autologhe e non autologhe con conseguente variare di espressività sul piano fisiopatologico e clinico. 


\section{BIBLIOGRAFIA}

1. Abulafia-Rapid R, Gillis D, Yosef O, et al. T cells and autoantibodies to human Hsp70 in type 1 diabetes in children. Autoimmun 2003; 20: 313-21.

2. Andrie R, Braun P, Welsch U, et al. Chlamydial and human heat shock protein 60 homologues in acute coronary syndromes. Z Kardiol 2003; 92: 455-8.

3. Anzola J, Luft BJ, Gorgone G, et al. Borrelia burgdorferi Hsp70 homolog: characterization of an immunoreactive stress protein. Infect Immun 1992; 60: 3704-13.

4. Becker LA, Cetin MS, Hutkins RW, Benson AK. Identification of the gene encoding the alternative sigma factor $\sigma^{\mathrm{B}}$ from Listeria monocytogenes and its role in osmotolerance. J Bacteriol 1998; 180: 4547-5.

5. Beinmet K, Soderstrom K, Jindal S, et al. Induction of heat shock protein 60 expression in human monocytic cell lines infected with Mycobacterium leprae. Infect Immun 1996; 64: 4356-8.

6. Betsou F, Sueur JM, Orfila J. Serological investigation of Chlamydia trachomatis heat shock protein 10 . Infect Immun 1999; 67: 5243-6.

7. Burns DL, Gould-Kostka J, Kessel M, Arciniega JL. Purification and immunological characterization of a GroEL-like protein from Bordetella pertussis. Infect Immun 1991; 59: 1417-22.

8. Cerrone MC, Ma JJ, Stephens RS. Cloning and sequence of the gene for heat shock protein 60 from Chlamydia trachomatis and immunological reactivity of the protein. Infect Immun 1991; 59: 79-90.

9. Craig EA, Gambill BD, Nelson RJ. Heat shock proteins: molecular chaperones of protein biogenesis. Microbiol Rev 1993; 57: 402-14.

10. Danilition SL, Maclean IW, Peeling R, et al. The 75kilodalton protein of Chlamydia trachomatis: a member of the heat shoch protein 70 family. Infect Immun 1990; 58: 189-96.

11. Domeika M, Domeika K, Paavonen J, Mardh PA. Humoral immune response to conserved epitopes of Chlamydia trachomatis and human $60-\mathrm{kDa}$ heat shock protein in women with pelvic inflammatory disease. J Infect Dis 1998; 177: 714-9.

12. Dominguez-Lopez ML, Burgos-Vargas R, GaliciaSerrano H, et al. IgG antibodies to enterobacteria 60 $\mathrm{kDa}$ heat shock protens in sera of HLA-B27 positive ankilosing spondilytis patients. Scand J Rheumatol 2002; 31: 260-5.

13. Dunn BE, Cohen H, Blaser MJ. Helicobacter pylori. Clin Microbiol Rev 1997; 10: 720-41.

14. Ensgraber M, Loos M. A 66-kilodalton heat shock protein of Salmonella typhimurium is responsible for binding of the bacterium to intestinal mucus. Infect Immun 1992; 60: 3072-8.

15. Fernandez RC, Logan SM, Lee SHS, Hoffman PS. Elevated levels of Legionella pneumophila stress protein Hsp60. Early in infection of human monocytes and L929 cells correlate with virulence. Infect Immun 1996; 64: 1968-76.

16. Garduno RA, Faulkner G, Trevors MA, et al. Immunolocalization of Hsp60 in Legionella pneumophila. J Bacteriol 1998; 180: 505-13.

17. Goulhen F, Hafezi A, Uitto VJ, et al. Subcellular localization and cytotoxic activity of the GroEL-like protein isolated from Actinobacillus actinomycetemcomitans. Infect Immun 1998; 66: 5307-13.

18. Habich C, Kempe K, Van der Zee R, et al. Different heat shock protein 60 species share pro-inflammatory activity but non binding sites on macrophages. FEBS
Lett 2003; 533: 105-9.

19. Hoffman P, Houston L, Butler CA. Legionella pneumophila htp-AB heat shock operon: nucleotide sequence and expression of the 60-kilodalton antigen in L. pneumophila - infected cells. Infect Immun 1990; 58: 3380-7.

20. Huittinen T, Leinonen M, Tenkanen L, et al. Synergistic effect of persistent Chlamydia pneumoniae infection autoimmunity and inflammation on coronary risk. Circulation 2003; 107: 2566-70.

21. Joo DM, Nolte A, Calendar R, et al. Multiple regions of the Escherichia coli heat shock transcription factor $\sigma 32$ determine core RNA polymerase binding specificity. J Bacteriol 1998; 180: 506-31.

22. Kwock AY, Su SC, Reynolds RP, et al. Species identification and phylogenetic relationships base on Hsp60 gene sequences within the genus Staphylococcus. Int J Syst Bacteriol 1999; 49: 1181-92.

23. Lamb DJ, El-Sankary W, Ferns GA. Molecular mimicry in atherosclerosis: a role for heat shock proteins in immunisation. Atherosclerosis 2003; 167: 177-85.

24. Lee A, Fox J, Hazell S. Pathogenicity of Helicobacter pylori: a perspective. Infect Immun 1993; 61:1601-10.

25. Lewthwaite J, Coates A, Tormay P, et al. Mycobacterium tuberculosis chaperonin 60.1 is a more potent cytokine stimulator than chaperonin 60.2 (Hsp65) and contains a CD14-binding domain. Infect Immun 2001; 69: 7349-55.

26. Lin J, Adams G, Ficht TA. Immunological response to the Brucella abortus GroEL homolog. Infect Immun 1996; 64: 4396-4400.

27. Lo WF, Woods AS, DeCloux A, et al. Moleculari mimicry mediatd by MHC class Ib molecules after infection with gram-negative pathogens. Nat Med 2000; 6: 215-8.

28. Mahdi OS, Horne BD, Mullen K, et al. Serum immunoglobulin $G$ antibodies to chlamydial heat shock protein 60 but not to human and bacterial homologs are associatd with coronary artery disease. Circulation 2002; 106: 1659-63.

29. Malago JJ, Koninks JF, Ovelgonne $\mathrm{HH}$, et al. Espression levels of heat shock proteins in enterocytelike Caco cells after exposure to Salmonella enteritidis. Cell Stress Chaperones 2003; 8: 194-203.

30. Mistry Y, Young DB, Mukherjee R. Hsp70 synthesis in Schwann cells in response to heat shock and infection with Mycobacterium leprae. Infect Immun 1992; 60: 3105-10.

31. Nielsen KL, Mc Lennan L, Masters M, Cowan NJ. A single ring mitochondrial chaperonin (Hsp60-Hsp10) can substitute for GroEL-GroES in vitro. J Bacteriol 1999; 181: 5871-5.

32. Parsons LM, Limberger LJ, Shayegani M. Alterations in levels of DnaK and GroEL result in diminished survival and adherence of stressed Haemophylus ducreyi. Infect Immun 1997; 65: 2413-9.

33. Phadnis SH, Parlow MH, Levy M, et al. Surface localization of Helicobacter pylori urease and heat shock protein homolog requires bacterial autolysis. Infect Immun 1996; 64: 905-12.

34. Pockley G. Heat shock proteins as regulators of the immune response. Lancet 2003; 362: 469-76.

35. Ritossa FA. A new puffing pattern induced by temperature shock and DNP in Drosophila. Experientia 1962; 18: 571 .

36. Sipos A, Klocke M, Frosch M. Cloning and sequencing of the genus coding for the 10 and $60 \mathrm{kDa}$ heat shock protein from Pseudomonas aeruginosa and mapping of a species-specific epitope. Infect Immun 1991; 59: 3219-26. 
37. Steinmetz I, Rheinheimer C, Hubner I, BitterSuermann D. Genus-specific epitope on the 60-kilodalton Legionella heat shock protein recognized by a monoclonal antibody. J Clin Microbiol 1991; 29: 346-54.

38. Stover CK, Marana DP, Dasch GA, Oaks EV. Molecular cloning and sequence analysis of the Sta58 antigen gene of Rickettsia tsutsugamushi: sequence homology and antigenic comparison of Sta58 to the 60-hilodalton family of stress proteins. Infect Immun 1990; 58: 1360-8.

39. Thies FL, Karch H, Hartung HP, Giegerich G. Cloning and expression of the dnaK gene of Campylobacter jejuni and antigenicity of heat shock protein 70 . Infect Immun 1999; 67: 1194-200.

40. Tissières A, Mitchell HK, Tracy U. Protein synthesis in salivary glands of Drosophila melanogaster: Relation to chromosome puffs. J Mol Biol 1974; 84: 389-98.

41. Wick G, Knoflach M, Xu Q. Autoimmune and inflammatory mechanisms in atherosclerosis. Ann Rev Immunol 2004; 22: 361-403.

42. Woods ML, Bonfiglioli R, McGee ZA, Georgopoulos C. Synthesis of a select group of proteins by Neisseria gonorrhoeae in response to thermal stress. Infect Immun 1990; 58: 719-25.

43. Yi Y, Yang X, Brunham RC. Autoimmunity to heat shock protein 60 and antigen-specific production of interleukin-10. Infect Immun 1997; 65: 1669-74.

44. Young DB, Garbe TR. Heat shock proteins and antigens of Mycobacterium tuberculosis. Infect Immun 1991; 59: 3086-93.

45. Yuan Y, Lyng K, Zhang YX, et al. Monoclonal antibodies define genus-specific, species-specific, and cross-reactive epitopes of the chlamydial 60 kilodalton heat shock protein (Hsp60): specific immunodetection and purification of chlamydial Hsp60. Infect Immun 1992; 60: 2288-96.

46. Zhang Y, Ohashi N, Rikihisa Y. Cloning of the heat shock protein 70 (Hsp70) gene of Ehrlichia sennetsu and differential expression of Hsp70 and Hsp60 mRNA after temperature upshift. Infect Immun 1998; 66: 3106-12. 